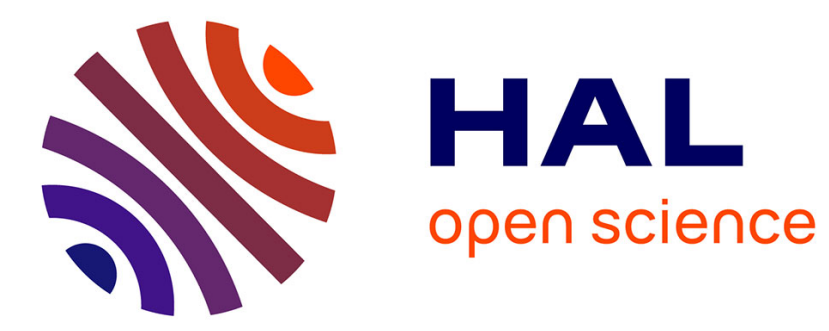

\title{
Phase change material-containing mesoporous zeolite composite for adsorption heat recovery
}

Jihye Choi, Valentin Valtchev, Masahiko Moteki, Masaru Ogura

\section{To cite this version:}

Jihye Choi, Valentin Valtchev, Masahiko Moteki, Masaru Ogura. Phase change material-containing mesoporous zeolite composite for adsorption heat recovery. Advanced Materials Interfaces, 2020. hal-03035395

\section{HAL Id: hal-03035395}

\section{https://hal-normandie-univ.archives-ouvertes.fr/hal-03035395}

Submitted on 2 Dec 2020

HAL is a multi-disciplinary open access archive for the deposit and dissemination of scientific research documents, whether they are published or not. The documents may come from teaching and research institutions in France or abroad, or from public or private research centers.
L'archive ouverte pluridisciplinaire HAL, est destinée au dépôt et à la diffusion de documents scientifiques de niveau recherche, publiés ou non, émanant des établissements d'enseignement et de recherche français ou étrangers, des laboratoires publics ou privés. 


\title{
Phase change material-containing mesoporous zeolite composite for adsorption heat recovery
}

\author{
Jihye Choi ${ }^{1}$, Valentin Valtchev ${ }^{2}$, Masahiko Moteki ${ }^{1}$, Masaru Ogura* ${ }^{1}$ \\ ${ }^{1}$ Institute of Industrial Science, The University of Tokyo, Komaba 4-6-1, Meguro-ku, Tokyo, Japan \\ ${ }^{2}$ Normandie Univ, ENSICAEN, UNICAEN, CNRS, Laboratoire Catalyse et Spectrochimie, 6 Boulevard Marechal Juin, \\ 14050 Caen, France \\ *E-mail: oguram@iis.u-tokyo.ac.jp
}

\begin{abstract}
A mesoporous zeolite is proposed as a phase change material (PCM) carrier. Owing to its physicochemical properties, the PCM-containing adsorbent has dual functions, namely simultaneous adsorption, and heat storage. In this study, the mesoporous chabazite-type zeolite SSZ-13, was prepared under various conditions to obtain optimized mesoporous SSZ13 (MSSZ-13) as a PCM-containing adsorbent. The phase change material was then successfully introduced in the created mesopore space through vapor phase synthesis, and the micropores could be utilized as an adsorption field. The efficiency of the as-prepared PCMcontaining MSSZ-13 was evaluated by a dehumidification process. The afforded experimental results showed suppressed bed temperature ramping, owing to the recovery of the heat generated upon $\mathrm{H}_{2} \mathrm{O}$ adsorption through the PCM. Moreover, the relative amount of adsorbed water increased at the initial period of time-on-stream of the contacting humid fluid.
\end{abstract}

\section{Introduction}

Zeolite is a crystalline microporous material composed of silica and alumina. It is one of the representative catalysts that has achieved industrial application, owing to its high surface area, molecular shape-selectivity, high thermal and chemical stabilities, and chemical properties that can be controlled via chemical composition and ion-exchangeability tuning ${ }^{1,2}$. On the other hand, molecular diffusion, pore blocking, and deactivation of the active sites inside the small micropores limit diffusivity through this material ${ }^{1,3}$. One method to mitigate this limitation is to introduce larger pores in the zeolite crystal ${ }^{3}$. This type of zeolite material, which possesses the natures derived from both micro- and mesopores, is called mesoporous or hierarchical zeolite ${ }^{4-6}$. There are several methods to introduce mesopores in zeolite crystals, including acid leaching ${ }^{7,8}$, alkaline leaching ${ }^{9,10}$, and fluoride etching ${ }^{11-13}$. A method using a surfactant as a template for introducing mesopores in zeolite has also been reported $^{14,15}$.

Owing to the above-mentioned advantages, zeolite is also widely used as an adsorbent in purification and separation processes ${ }^{2}$. In the adsorption process, the adsorption performance of the material is significantly affected by the increased adsorbent temperature due to the released heat of adsorption ${ }^{16}$. Therefore, methods to improve adsorption heat removal have been extensively studied ${ }^{17-20}$. Particularly, in short-time adsorption processes, the period for cooling the adsorbent is extremely short and hence, rapid removal of the heat of adsorption is essential to avoid degradation of the adsorption performance. Gas storage, adsorption heat pump (AHP), and pressure swing adsorption (PSA) processes are well-known examples of 
short-time adsorption processes ${ }^{17-20}$.

In many adsorption processes, the heat of adsorption is generally removed by a heat exchanger, a system that cools a process by transferring the released heat to heat transfer fluids. Another method uses the latent heat of a phase-change material (PCM) to remove the released heat of adsorption. One of the advantages of this latter method is that it allows the storage of the thermal energy within a narrow temperature range and its subsequent reutilization. Thus, the application of a PCM capsule or PCM-containing metallic void to the adsorption process prevents the drastic increase in temperature usually observed in this process $^{19,21}$. As a possible target of the PCM-containing adsorbent, a short-time adsorption process is considered, such as gas storage, PSA, and AHP, where adsorption heat recovery is required. Therefore, hydrogen, carbon dioxide, and water should be removed, via adsorption, by the PCM-containing adsorbent. In previous research using PCM in the adsorption process, heat transfer was reported to occur in two steps: the first one is from the adsorption site to the heat transfer medium, and the second one is from the medium to the PCM. A gaseous compound such as dried air or nitrogen is commonly employed as the heat transfer medium in the adsorption process. However, the slow heat transfer due to the small heat capacity of gaseous matter has been a major limitation for using PCM in adsorption processes ${ }^{22}$.

Recently, we have proposed a new concept for the application of a PCM-containing adsorbent $^{22}$. By inserting a PCM molecule directly into the pores of the adsorbent, adsorption and adsorption heat recovery occur simultaneously in the same adsorbent particles. The inserted PCM is strongly confined in the porous space by the surface tension and thus, the amount of PCM leaked from the adsorbent pores during the operation is negligible. In this PCM-containing adsorbent concept, the heat of adsorption can be effectively removed by the confined PCM, owing to the extremely short heat transfer distance, without passing through the heat transfer medium. Moreover, the presence of the PCM increases the specific heat capacity of the material. This indicates that a PCM-containing adsorbent has the advantages of both latent and sensible heat storage. Therefore, rapid removal of the heat of adsorption can be expected by applying a PCM-containing adsorbent. Additionally, PCM can be homogenously distributed in the adsorption bed, as there is no density difference between the pelletized PCM-containing adsorbents. These facts indicate that an isothermal-adiabatic adsorption process can be realized by developing a PCM-containing adsorbent. Moreover, the adsorption performance of the short-time adsorption process could be upgraded simultaneously.

A specific concept of coexisting PCM and adsorbate inside the adsorbent is necessary to elaborate such a PCM-containing adsorbent. The host container for the PCM molecules should possess a large vacant space where a phase change, from solid to liquid, occurs. Furthermore, separation of the PCM and adsorbate inside the adsorbent is required in the specific concept to prevent the adsorbate from dissolving in the liquified PCM. We have reported PCM-containing mesoporous silica SBA-15 to exemplify a PCM-containing adsorbent. In this material, the mesoporous spaces are utilized as a heat storage field by the PCM, while the vacant spaces, where no PCM is present in the mesopores or external surface, are utilized as adsorption sites. Notably, PCM-containing SBA-15 exhibited isothermaladiabatic behavior and an improved adsorption performance during the initial few minutes of the dehumidifying process. This indicates the achievement of the developing objectives of the PCM-containing adsorbent. However, PCM-containing SBA-15 is just one example of a 
specific concept for PCM-containing adsorbent.

Herein, we propose the PCM-containing mesoporous zeolite SSZ-13 as another specific concept for the PCM-containing adsorbent. A schematic image of the concept, in which the adsorption site is located in the micropore and the mesoporous space is used as a heat storage field, is presented in Figure 1. One advantage of this concept is that the separation of the PCM and adsorbate in the zeolite crystal can be achieved by the size difference between the PCM molecule and adsorbate. Thus, by selecting a PCM molecule with a larger size than the micropore diameter, the PCM could only be accommodated in the mesopores. Using the data from our previous report ${ }^{23}$, we selected the chabazite (CHA)-type high-silica zeolite SSZ-13 with an ultra-small micropore $(\sim 0.4 \mathrm{~nm})$ as the zeolite material, while paraffin was selected as the representative organic PCM. This as-synthesized PCM-containing mesoporous SSZ-13 (MSSZ-13) was then evaluated by monitoring the bed temperature and amount of adsorbed water in a fixed-bed dehumidifying adsorption process.

\section{Experimental}

\subsection{Preparation of MSSZ-13}

Two different methods were conducted to introduce the mesopores in zeolite SSZ-13, one using ammonium fluoride $\left(\mathrm{NH}_{4} \mathrm{~F}\right)$ and the other sodium hydroxide $(\mathrm{NaOH})$ aqueous solutions. The parent zeolite SSZ-13 $\left(\mathrm{SiO}_{2} / \mathrm{Al}_{2} \mathrm{O}_{3}=18\right)$ was supplied by a reference zeolite project of the Japan Zeolite Association.

Fluoride etching treatment of SSZ-13 was conducted in an ultrasonic (US) bath. Specifically, $1 \mathrm{~g} \mathrm{NH}_{4}{ }^{+}$ion-exchanged SSZ-13 was added to $20 \mathrm{~g}$ of $40 \mathrm{wt} \% \mathrm{NH}_{4} \mathrm{~F}$ solution. The mixed slurry was subjected to different periods (20-120 min) of ultrasonic treatment at 25 and $40{ }^{\circ} \mathrm{C}$. The slurry was then filtered and washed threefold with deionized water $\left(60{ }^{\circ} \mathrm{C}\right.$, $500 \mathrm{~mL}$ ). Subsequently, the filtrate was collected and dried overnight in air. The treatment was also carried out using double the zeolite/solution ratio $\left(1 \mathrm{~g} \mathrm{NH}_{4}{ }^{+}-\mathrm{SSZ}-13\right.$ zeolite in $40 \mathrm{~g}$ of $40 \mathrm{wt} \% \mathrm{NH}_{4} \mathrm{~F}$ solution), whereby the ultrasonic bath temperature was raised from 25 to 40 and $55^{\circ} \mathrm{C}$, for 1 and $2 \mathrm{~h}$.

Alkaline leaching treatment of SSZ-13 was conducted hydrothermally. Thus, $1 \mathrm{~g} \mathrm{Na}^{+}$ionexchanged SSZ-13 was added to $30 \mathrm{~mL}$ of $0.6 \mathrm{M} \mathrm{NaOH}$ solution, and the slurry was stirred at $65{ }^{\circ} \mathrm{C}$ for several minutes $(5,15,45$, and $90 \mathrm{~min})$ at a stirring speed of $400 \mathrm{rpm}$. After several minutes, the solution was filtered and washed threefold with deionized water $\left(60{ }^{\circ} \mathrm{C}, 500 \mathrm{~mL}\right)$. The filtrate was then allowed to dry overnight.

All the products prepared according to the above-described methods were labeled according to their preparation conditions, as summarized in Table 1. Finally, the prepared products were characterized by X-ray diffraction (XRD), scanning electron microscopy (SEM), and $\mathrm{N}_{2}$ adsorption techniques.

\subsection{Preparation and characterization of PCM-containing MSSZ-13}

Paraffin (C22, docosane) was inserted into the mesopores of as-prepared MSSZ-13 (Section 2.1) via a vapor transportation method ${ }^{24}$. Thus, MSSZ-13, was dried at $100{ }^{\circ} \mathrm{C}$ overnight and placed in an encapsulated desiccator. The PCM solid/liquid was separately placed in the same desiccator. Next, the desiccator was evacuated, sealed, and maintained at $150{ }^{\circ} \mathrm{C}$ in an air oven for several hours overnight until the adsorption of vaporized paraffin on 
MSSZ-13 reached equilibrium. The ratio of the volume by paraffin inside the mesopores, $w$, was calculated by weighting the sample before and after inserting the paraffin, as follows:

$$
w=\frac{100\left(m_{a}-m_{b}\right)}{\rho m_{b} V_{t}}
$$

where $m_{a}$ represents the weight of PCM-containing SSZ-13, $m_{b}$ represents the weight of the dried MSSZ-13 before PCM vapor treatment, $\rho$ represents the PCM density, and $V_{t}$ represents the total pore volume of MSSZ-13 measured by $\mathrm{N}_{2}$ adsorption. The value $w$ can

be considered as the pore filling ratio of the PCM inside the mesopores.

The porous characteristics of the materials were measured by $\mathrm{N}_{2}$ adsorption, while the melting point and enthalpy of phase changes of the PCM were measured by differential scanning calorimetry (DSC8230 Thermoplus, Rigaku). Each DSC measurement was repeated fivefold to determine whether any liquefied PCM leaked from the composite material. Additionally, water vapor adsorption isotherms were collected at different temperatures (293-318 K) to verify that the adsorption sites were still present after PCM insertion.

\subsection{Dehumidifying adsorption process}

Dehumidification using a packed bed of PCM-containing MSSZ-13 was carried out to evaluate the prepared PCM-containing MSSZ-13. As a reference, the dehumidification process was also performed using the parent SSZ-13. Thermal conductivity detection (TCD, GC8A, Shimadzu) was employed to detect water vapor throughout the packed bed of adsorption material. The pelletized adsorbent was sieved in the range $2-4 \mathrm{~mm}$ and then packed in the bed. Nitrogen was supplied by two different lines, one directly connected to the adsorbent column and the other connected to a bubbler in a water bath $\left(50-80{ }^{\circ} \mathrm{C}\right)$ to create humid nitrogen. The humidified nitrogen was allowed to flow through the adsorbent bed column. The flow rate of each line was controlled by a flowmeter at $100 \mathrm{~cm}^{3} / \mathrm{min}$. In all the experiments, the relative humidity at the inlet was fixed at $50 \%$, while the initial temperature of the adsorption bed was maintained at $29.5^{\circ} \mathrm{C}$. The adsorbent bed column was lagged with styrofoam to ensure adiabatic conditions. The temperature of the adsorbent bed was measured at two points, namely near the inlet and outlet of the column, using a K-type thermocouple. The length of the adsorbent bed was $2 \mathrm{~cm}$, and the diameter of the bed was $1 \mathrm{~cm}$., The pretreatment of the adsorbent was performed at $60^{\circ} \mathrm{C}$ for $3 \mathrm{~h}$ under dried nitrogen flow.

\section{Results and discussion}

\subsection{Preparation of MSSZ-13}

For the primary purpose of developing a PCM-containing adsorbent, a high storage capacity for latent heat is preferable. The storage capacity is determined by the enthalpy of fusion and the amount of PCM inserted inside the matter, which in turn are determined by the size and volume of the container space. Additionally, in the concept of the PCM-containing MSSZ-13, the microporous space is necessarily utilized for adsorption, and thus, preservation of the micropore volume of the SSZ-13 zeolite is another important factor for the establishment of the concept. Hence, for the development of a PCM-containing adsorbent, the MSSZ-13 must have a large mesopore diameter and volume, while maintaining the 
microporous volume. Thus, in this study, MSSZ-13 was prepared under various conditions to obtain the optimal MSSZ-13.

\subsubsection{Effect of the treatment solution on the preparation}

Aqueous $\mathrm{NH}_{4} \mathrm{~F}$ and $\mathrm{NaOH}$ solutions were applied to the respective etching and leaching treatments employed in this study. The products prepared by fluoride etching and alkali leaching for various treatment periods were characterized by XRD, $\mathrm{N}_{2}$ adsorption, and SEM techniques. The XRD patterns of the MSSZ-13 samples prepared by fluoride etching are depicted in Figure 2 (a), while those of the MSSZ-13 prepared by alkali leaching are presented in Figure 2 (b). The results revealed that the XRD patterns of all the MSSZ-13 samples were identical to those observed for SSZ-13. This indicates that the crystalline microporous structure of SSZ-13 is still retained after fluoride etching and alkali leaching. However, for both processes, the intensities of the XRD peaks gradually decreased with prolonged treatment. This suggests that both methods somewhat destroy the crystalline structure of the materials. Moreover, the intensities of the products formed by alkali leaching exhibited a greater decrease than those of the products prepared by fluoride etching. It is generally accepted that dissolved $\mathrm{NH}_{4} \mathrm{~F}$ provides $\mathrm{HF}_{2}{ }^{-}$ions, which indiscriminately dissolve both the alumina and silica in the zeolite material ${ }^{12,13}$. On the other hand, $\mathrm{NaOH}$ only extracts silica from the framework ${ }^{9}$. Consequently, the preferential silica extraction from a silica-rich materialas SSZ-13 ( $\mathrm{Si} / \mathrm{Al}$ ratio $\sim 9$ ) leads to substantial crystallinity loss.

The nitrogen adsorption isotherms and their derived pore size distributions (BJH method) are respectively displayed in Figures 3 (a) and (b) for the products prepared by fluoride etching and in Figures 3 (c) and (d) for those obtained by alkali leaching. Additionally, the values of the micro- and mesopore volumes and average pore diameters are summarized in Table 2, and clearly demonstrate that both treatments successfully introduced mesopores. Specifically, in the samples prepared by fluoride etching, a gradual increase in the mesopore volume accompanied by a slight decrease in the micropore volume was observed with prolonged treatment. Comparatively, the products prepared by alkali leaching displayed a significant increase in the mesopore volume and a marked decrease in the micropore volume with increasing treatment time. This decrease in the micropore volume is in good agreement with the decrease in the crystallinity of zeolite, as illustrated in Figure 2. Notably, unlike the other samples, sample HT90 displayed a reduction in both the micro- and mesopore volumes with increasing treatment time. Compared to the products obtained by fluoride etching, the decrease in micropore volume was more significant than the increase in the mesopore volume for the products prepared by alkali leaching. For example, after $15 \mathrm{~min}$ alkali leaching, the sample (HT15) presented a 77\% decrease from the original micropore volume and a mesopore volume of $0.15 \mathrm{~cm}^{3} / \mathrm{g}$. On the other hand, no decrease in micropore volume and a mesopore volume of $0.10 \mathrm{~cm}^{3} / \mathrm{g}$ was observed after $20 \mathrm{~min}$ of fluoride etching (US20). Relatively wide pore size distributions, with mesopore sizes in the range of 2-50 $\mathrm{nm}$, were observed for the products afforded by both techniques. This indicates that the mesopores were not uniformly introduced in the SSZ-13 material applied in this investigation.

The morphology features of SSZ-13 and the as-prepared MSSZ-13 are illustrated in Figure 4. As can be seen in Figure 4 (A), the parent SSZ-13 exhibits rectangular-shaped particles. The MSSZ-13 samples [Figures 4 (B)-(I)] displayed rectangular morphologies similar to those of the parent SSZ-13, even after fluoride etching and alkali leaching treatment. 
However, treatment slightly changed the edges of the particles, making them rounder in shape. Moreover, in the SEM image of HT90 [Figure 4 (I)], a crack was observed at the center of the particles, owing to the severe leaching. Such a drastic change in the morphology of the zeolite particles and the generation of surface cracks were also reported in the literature for ZSM-5 zeolite treated with a highly alkaline solution ${ }^{10}$.

At this stage, we could confirm that both methods were successful in introducing a large mesopore inside the microporous zeolite SSZ-13 particles. It was further suggested that alkali leaching is a destructive method against SSZ-13 zeolite, which resulted in a significant decrease in the micropore volume and crystallinity, compared to those attained with fluoride etching. As already mentioned, it is important to introduce a large mesopore while preserving the micropore of the mother zeolite crystal to be appropriate as a PCM-containing adsorbent. Therefore, fluoride etching was considered as the optimal method for the creation of MSSZ13 .

\subsubsection{Effect of temperature on the preparation}

The MSSZ-13 samples, namely US20, US40, US90, and US120, were prepared at $25^{\circ} \mathrm{C} \mathrm{w}$ ith a zeolite/solution ratio of $1 / 20$ (Table 1). To investigate the effect of the treatment temperature on the material, fluoride etching was carried out at higher temperatures of $40 \mathrm{a}$ nd $55{ }^{\circ} \mathrm{C}$. Additionally, the zeolite/solution ratio was doubled to obtain a larger mesopore volume, while the concentration of the fluoride solution was unchanged. The products prepared under these slightly more severe conditions were named according to their treatment temperature and time (US40d-1H, US40d-2H, US55d-1H, and US55d-2H; Table 1). The XRD patterns of the etched SSZ-13 samples prepared under the higher temperatures are presented in Figure 5. The same XRD patterns were observed for the products that originated from SSZ-13, indicating that the microporous crystalline structure of SSZ-13 was maintained after fluoride etching. However, with the increase in the treatment temperature and time, the intensities of the XRD patterns were drastically decreased. This indicates that the crystallinity of the SSZ-13 material decreases with increasing treatment temperature and time, owing to the more severe etching conditions.

The nitrogen adsorption-desorption isotherms and pore size distribution curves of the etched SSZ-13 samples are depicted in Figures 6 (a) and (b), respectively, while the physicochemical properties of etched materials are summarized in Table 3. A significant mesopore generation was observed in all the products. With the increase in the treatment temperature from $25^{\circ} \mathrm{C}$ to 40 and $55^{\circ} \mathrm{C}$ (i.e., from US60 to US40d-1H and US55d-1H), the mesopore volume largely increased. On the other hand, a further increase in the treatment time from 1 to $2 \mathrm{~h}$ led to a decrease in the mesopore volume, which seems to correlate with the results observed for HT90. Generally, with the increase in mesopore volume, the micropore volume decreased according to the treatment temperature and period, as summarized in Figure 7. However, for US40d-2H and US55d-2H, the mesoporous and microporous volumes decreased simultaneously. The pore size distribution became broader in the etched products. Similar to the changes in the mesopore volume, the mesopore diameter also decreased with increasing treatment time. This is possibly due to the formation of macropores that could not be detected by nitrogen adsorption or the presence of a relatively small amount of mesopores as a result of crystal destruction.

The scanning electron micrographs of the as-prepared MSSZ-13 samples are illustrated in Figure 8. All the MSSZ-13 materials obtained in this study displayed a morphology similar to 
that of the parent SSZ-13 [Figure 4 (A)], but with a relatively rounder shape along with the harder fluoride etching.

At this stage of the investigation, fluoride etching was determined as an effective method, over alkali leaching, to introduce mesopores in SSZ-13 while preserving the original micropores of SSZ-13. It is generally preferable to introduce a mesopore large enough to occlude the PCM, which corresponds to the heat storage capacity of perfect absorption of the released heat of adsorption. The latent heat storage capacity is determined from the amount of occluded PCM, since the enthalpy of fusion is a physical property of this material. A mesopore volume dozens of times larger than the micropore volume is required to completely absorb the released heat of adsorption, mainly due to the large quantity of the heat. On the other hand, preservation of the micropore volume while introducing mesopores is also important to maintain the adsorption capacity. A trade-off between maintaining the micropore volume and increasing the mesopore volume was, therefore, necessary, as illustrated in Figure 8. Thus, MSSZ-13 with a mesopore volume at least three- to fourfold larger than the micropore volume could be attained to utilize the material as a PCM container. Possible application of the PCM-containing adsorbent is by a rapid adsorption process, which runs before the adsorption system reaches adsorption equilibrium. Among the MSSZ-13 materials prepared in this study, US40d-1H displayed less crystallinity degradation, a large mesopore volume, and a relatively smaller decrease in micropore volume. Therefore, this material was selected for further investigation to determine its potential as a PCM-containing MSSZ-13.

The surface scanning electron micrographs of US40d-1H (Figure 9) confirm the introduction of non-uniform mesopores in this material. Indeed, various mesopore shapes were observed, including cheese-like holes ([Figures 9 (A) and (B)], honeycomb-like holes [Figure $9(\mathrm{C})$ ], and large crater-like holes [Figure 9 (D)] that indicated the introduction of not only mesopores but also macropores in some parts of the crystal.

\subsection{Characterization of the as-prepared PCM-containing MSSZ-13 materials}

In this study, paraffin was used as the PCM, which was inserted into the mesopores of MSSZ-13 via a vapor transportation method. First, it was necessary to confirm that paraffin could be used as the PCM in the concept of composite PCM-containing MSSZ-13. To utilize the microporous space as an adsorption field, the PCM molecule must be larger than the micropore size of MSSZ-13. The nitrogen adsorption isotherms of SSZ-13 and paraffinloaded SSZ-13 are demonstrated in Figure 10. Both porous materials displayed a drastic increase in the adsorbed amount of nitrogen in the low $\mathrm{P} / \mathrm{P}_{0}$ range, with no noticeable differences between them. This uptake of nitrogen in the $\mathrm{P} / \mathrm{P}_{0}$ range below 0.1 is derived from the nitrogen adsorbed in the microporous space. If paraffin were able to access the micropore, the microporous space would be occupied by the confined paraffin, and the nitrogen uptake would not be observed. Hence, the results confirmed that paraffin did not access the SSZ-13 micropores and thus the prepared material could be used as the PCM for this study.

The measured nitrogen adsorption isotherms of the MSSZ-13 and PCM-containing MSSZ13 materials are presented in Figure 11. At the same time, the changes in the porous structure properties before and after PCM insertion are listed in Table 4. By inserting PCM into MSSZ13, the mesopore volume was significantly decreased compared to that of the parent MSSZ13. This indicated that PCM was successfully introduced solely into the mesopores of SSZ-13. However, nearly $40 \%$ of the MSSZ-13 micropore volume was simultaneously decreased. As confirmed in Figure 10, PCM does not occupy the microporous space of SSZ-13. Instead, PCM occupies the mesoporous space of MSSZ-13 and supposedly partially blocks diffusion 
to the micropores. Hence, separation of the adsorption site (micropores) and heat storage field of paraffin (phase change in the mesopores), was successfully achieved.

To investigate the adsorption properties of water vapor on SSZ-13 and PCM-containing MSSZ-13, $\mathrm{H}_{2} \mathrm{O}$ adsorption isotherms were measured at three different temperatures for SSZ13 and at six different temperatures for PCM-containing MSSZ-13, as illustrated in Figure 12. Under the tested conditions, with $\mathrm{x}$-axial relative pressure, the isotherms indicated that there was no apparent temperature dependency of the adsorption on SSZ-13 and PCM-containing MSSZ-13. For SSZ-13, the $\mathrm{H}_{2} \mathrm{O}$ adsorption isotherm was a Langmuir type I isotherm. On the other hand, PCM-containing SSZ-13 presented a Langmuir type I isotherm at the lower relative pressure range, while $\mathrm{H}_{2} \mathrm{O}$ condensation was observed at the higher pressure range at relative pressures above 0.8 . This indicated that not all the mesoporous spaces were filled with PCM. Thus, after the PCM insertion process, the amount of adsorbed water vapor decreased at relative pressures lower than 0.7 . This is because, as already discussed (Section 3.1.1), the micropore volume is reduced by fluoride etching, and some of the micropores become blocked after PCM insertion. However, even though a decrease in the amount of adsorbed $\mathrm{H}_{2} \mathrm{O}$ was detected, $\mathrm{H}_{2} \mathrm{O}$ adsorption sites were still clearly present in the prepared PCM-containing MSSZ-13.

\subsection{Evaluation of the phase change and heat recovery of PCM-containing MSSZ-13}

The melting properties and stability of the PCM inserted in MSSZ-13 were characterized by repeated DSC measurements. The phase change peak of the paraffin inserted in MSSZ-13 was observed (Figure 13), indicating the heat storage ability of the inserted paraffin. Moreover, the phase change (i.e., the phase change temperature and amount of heat recovery of the inserted paraffin) did not change during the repeated measurements, suggesting that the inserted PCM did not change physically or chemically. Moreover, the PCM did not seem to leak from the mesoporous space of MSSZ-13, even in the liquid state with thermal stability. The melting point of the inserted paraffin was $42.6^{\circ} \mathrm{C}$, while its enthalpy of fusion was 156 $\mathrm{J} / \mathrm{g}-\mathrm{PCM}$. These values were slightly lower than those of the original PCM $\left(43.0^{\circ} \mathrm{C}\right.$ and 237 $\mathrm{J} / \mathrm{g}-\mathrm{PCM}$, respectively), owing to the surface tension and interaction between the PCM molecules and the mesopore wall of MSSZ-13. These results confirmed that PCM-containing MSSZ-13 having dual functions of heat storage and adsorption was successfully prepared.

These functions on the prepared PCM-containing MSSZ-13 were evaluated by a dehumidification process. The objective of developing PCM-containing MSSZ-13 was to achieve a performance upgrade of such a thermal process through absorption heat recovery. Therefore, the effectiveness was evaluated from the change in the amount of adsorbed water within the initial period of time-on-stream and by the difference in the system temperature. The temperature of the adsorbent was measured near the inlet and outlet of the fixed bed, as illustrated in Figures 14 (a) and (b), respectively. Additionally, the amount of $\mathrm{H}_{2} \mathrm{O}$ adsorbed on the adsorbent was calculated from the changes in the $\mathrm{H}_{2} \mathrm{O}$ concentration, which were detected by TCD [Figure 14 (c)]. The amount of adsorbed water relative to the equilibrium amount of adsorbed water is presented in Figure 14 (d). The relative humidity of the fluid at the inlet was fixed at $50 \%$. Thus, the equilibrium amount of adsorbed water, $q^{*}$, corresponds to the amount of adsorbed water on PCM-containing MSSZ-13 when $P / P_{0}$ is 0.5 [Figure 12 (b)]. As illustrated in Figures 14 (a) and (b), regardless of the position in the bed (near the inlet or outlet), the rapid increase in the bed temperature at the initial adsorption period was suppressed by the coexistence with the PCM. This proves that the PCM inserted in the adsorbent works successfully as a heat storage material. The value of $q / q^{*}$ on PCM- 
containing MSSZ-13 [Figure 14 (d)] was higher than that on SSZ-13 during the initial 5 minutes after flowing the humid fluid. Therefore, the PCM-containing MSSZ-13 can successfully recover the heat of adsorption, thereby resulting in an increased apparent rate of adsorption, as shown in the results. On the other hand, even though successful heat recovery through PCM was achieved, the amount of adsorbed water on the PCM-containing MSSZ-13 was slightly smaller than that on SSZ-13 [Figure 14 (c)]. This might be derived from the decrease in $q^{*}$ after PCM insertion (Figure 13), which has a more significant effect on the amount of adsorbed $\mathrm{H}_{2} \mathrm{O}$ than the heat recovery through the PCM. Additionally, the temperature dependence of the adsorbed water on SSZ-13 is relatively small. Therefore, as future perspectives, the use of a more temperature-sensitive zeolite, such as SAPO- $34^{25}$, as the mesoporous zeolitic container and application of PCM-containing MSSZ-13 to a more exothermic adsorption process should be considered to achieve drastic performance improvement in the adsorption processes.

In summary, a PCM-containing MSSZ-13 having simultaneous adsorptive and heat storage properties in the same material was successfully prepared. Additionally, the as-prepared PCM-containing MSSZ-13 was proven as a valid concept for the PCM-containing adsorbent.

\section{Conclusions}

As a new concept of PCM-containing adsorbent, a PCM-in-MSSZ-13 composite material was proposed as an adsorbent possessing simultaneous adsorptive and heat storage abilities in the same particles. An MSSZ-13 material with a large mesopore volume was used as the host material for inserting PCM solely in the mesopore. The mesopore was created by a fluoride etching method. Thus, PCM-containing MSSZ-13 was successfully prepared, and its mesoand microporosities were confirmed. The mesoporous space was utilized as a heat storage field, while the microporous space, originating from SSZ-13, was utilized as an adsorption field. The experimental results of the as-prepared PCM-containing MSSZ-13 revealed suppressed bed temperature ramping and an increase in the relative amount of adsorbed water vapor, $q / q^{*}$. Therefore, it can be concluded that PCM-containing MSSZ-13 delivers an effective concept, namely that the performance can be upgraded through the heat adsorption recovery, and the composite can be another application for the utilization of mesoporous zeolite.

\section{Acknowledgement}

The authors would like to acknowledge Microtrac BEL Corporation for their support on the measurement of the $\mathrm{H}_{2} \mathrm{O}$ adsorption isotherms for a series of SSZ-13 materials. Observation of US40d-1H by surface SEM was conducted at the Advanced Characterization Nanotechnology Platform of the University of Tokyo, supported by "Nanotechnology Platform" of the Ministry of Education, Culture, Sports, Science and Technology (MEXT), Japan. MO would like to thank Editage (www.editage.com) for English language editing. V.V. acknowledges the financial support from the ANR-TOTAL Industrial Chair 'NanoClean Energy".

\section{References}


(1) Valtchev, V.; Mintova, S. Hierarchical Zeolites. MRS Bull. 2016, 41 (9), 689-693. https://doi.org/10.1557/mrs.2016.171.

(2) Davis, M. E. Zeolites and Molecular Sieves: Not Just Ordinary Catalysts. Ind. Eng. Chem. Res. 1991, 30 (8), 1675-1683.

https://doi.org/10.1021/ie00056a001.

(3) Holm, M. S.; Taarning, E.; Egeblad, K.; Christensen, C. H. Catalysis with Hierarchical Zeolites. Catal. Today 2011, 168 (1), 3-16.

https://doi.org/10.1016/j.cattod.2011.01.007.

(4) Koohsaryan, E.; Anbia, M. Nanosized and Hierarchical Zeolites: A Short Review. Chinese J. Catal. 2016, 37 (4), 447-467.

https://doi.org/10.1016/S1872-2067(15)61038-5.

(5) Hartmann, M. Hierarchical Zeolites: A Proven Strategy to Combine Shape Selectivity with Efficient Mass Transport. Angew. Chemie - Int. Ed. 2004, 43 (44), 5880-5882. https://doi.org/10.1002/anie.200460644.

(6) Tao, Y.; Kanoh, H.; Abrams, L.; Kaneko, K. Mesopore-Modified Zeolites: Preparation, Characterization, and Applications. Chem. Rev. 2006, 106 (3), 896-910. https://doi.org/10.1021/cr040204o.

(7) Yang, S.; Yu, C.; Yu, L.; Miao, S.; Zou, M.; Jin, C.; Zhang, D.; Xu, L.; Huang, S. Bridging Dealumination and Desilication for the Synthesis of Hierarchical MFI Zeolites. Angew. Chemie - Int. Ed. 2017, 56 (41), 12553-12556. https://doi.org/10.1002/anie.201706566.

(8) Shetti, V. N.; Kim, J.; Srivastava, R.; Choi, M.; Ryoo, R. Assessment of the Mesopore Wall Catalytic Activities of MFI Zeolite with Mesoporous/Microporous Hierarchical Structures. J. Catal. 2008, 254 (2), 296-303. https://doi.org/10.1016/j.jcat.2008.01.006.

(9) Sommer, L.; Mores, D.; Svelle, S.; Stöcker, M.; Weckhuysen, B. M.; Olsbye, U. Microporous and Mesoporous Materials Mesopore Formation in Zeolite H-SSZ-13 by Desilication with NaOH. Microporous Mesoporous Mater. 2010, 132 (3), 384-394. https://doi.org/10.1016/j.micromeso.2010.03.017.

(10) Ogura, M.; Shinomiya, S. -y.; Tateno, J.; Nara, Y.; Kikuchi, E.; Matsukata, M. Formation of Uniform Mesopores in ZSM-5 Zeolite through Treatment in Alkaline Solution. Chem. Lett. 2000, 29 (8), 882-883.

https://doi.org/10.1246/cl.2000.882.

(11) Du, S.; Chen, X.; Sun, Q.; Wang, N.; Jia, M.; Valtchev, V.; Yu, J. A Non-Chemically Selective Top-down Approach towards the Preparation of Hierarchical TS-1 Zeolites with Improved Oxidative Desulfurization Catalytic Performance. Chem. Commun. 2016, 52 (17), 3580-3583.

https://doi.org/10.1039/c5cc10232d.

(12) Qin, Z.; Melinte, G.; Gilson, J. P.; Jaber, M.; Bozhilov, K.; Boullay, P.; Mintova, S.; Ersen, O.; Valtchev, V. The Mosaic Structure of Zeolite Crystals. Angew. Chemie - Int. Ed. 2016, 55 (48), 15049-15052. 
https://doi.org/10.1002/anie.201608417.

(13) Chen, X.; Vicente, A.; Qin, Z.; Ruaux, V.; Gilson, J.-P.; Valtchev, V. The Preparation of Hierarchical SAPO-34 Crystals via Post-Synthesis Fluoride Etching. Chem.

Commun. 2016, 52 (17), 3512-3515.

https://doi.org/10.1039/c5cc09498d.

(14) Sachse, A.; García-Martínez, J. Surfactant-Templating of Zeolites: From Design to Application. Chem. Mater. 2017, 29 (9), 3827-3853.

https://doi.org/10.1021/acs.chemmater.7b00599.

(15) Garcia-Martinez, J.; Xiao, C.; Cychosz, K. A.; Li, K.; Wan, W.; Zou, X.; Thommes, M. Evidence of Intracrystalline Mesostructured Porosity in Zeolites by Advanced Gas Sorption, Electron Tomography and Rotation Electron Diffraction. ChemCatChem 2014, 6 (11), 3110-3115.

https://doi.org/10.1002/cctc.201402499.

(16) Hwang, K. S.; Jun, J. H.; Lee, W. K. Fixed-Bed Adsorption for Bulk Component System. Non-Equilibrium, Non-Isothermal and Non-Adiabatic Model. Chem. Eng. Sci. 1995, 50 (5), 813-825.

https://doi.org/10.1016/0009-2509(94)00433-R.

(17) Demir, H.; Mobedi, M.; Ülkü, S. A Review on Adsorption Heat Pump: Problems and Solutions. Renew. Sustain. Energy Rev. 2008, 12 (9), 2381-2403.

https://doi.org/10.1016/j.rser.2007.06.005.

(18) Wang, R. Z. Performance Improvement of Adsorption Cooling by Heat and Mass Recovery Operation. Int. J. Refrig. 2001, 24, 602-611.

https://doi.org/10.1016/S0140-7007(01)00004-4.

(19) Li, X.; Li, Y. Applications of Organic Phase Change Materials Embedded in Adsorbents for Controlling Heat Produced by Charging and Discharging Natural Gas. Adsorption 2015, 21, 383-389.

https://doi.org/10.1007/s10450-015-9678-4.

(20) Horstmeier, J. F.; Gomez Lopez, A.; Agar, D. W. Performance Improvement of Vacuum Swing Adsorption Processes for CO2 Removal with Integrated Phase Change Material. Int. J. Greenh. Gas Control 2016, 47, 364-375. https://doi.org/10.1016/j.ijggc.2016.02.013.

(21) Rady, M. A.; Huzayyin, A. S.; Arquis, E.; Monneyron, P.; Lebot, C.; Palomo, E. Study of Heat and Mass Transfer in a Dehumidifying Desiccant Bed with MacroEncapsulated Phase Change Materials. Renew. Energy 2009, 34, 718-726. https://doi.org/10.1016/j.renene.2008.04.038.

(22) Choi, J.; Yoshie, K.; Moteki, T.; Ogura, M. Theoretical Evaluation of an Organic Phase Change Material (PCM)-Inserted Dual-Functional Adsorbent for the Recovery of Heat of Adsorption. Ind. Eng. Chem. Res. 2019, 58 (23), 10114-10118. https://doi.org/10.1021/acs.iecr.9b00198.

(23) Choi, J.; Ogura, M. Mesoporous Zeolite for Use as Dual-functional Heat-storage 
Adsorbent. Chem. Lett. 2020, 49, 450-452.

https://doi.org/10.1246/cl.200073.

(24) Choi, J.; Fujita, H.; Ogura, M.; Sakoda, A. Confinement Effect on Enthalpy of Fusion and Melting Point of Organic Phase Change Materials in Cylindrical Nanospace of Mesoporous Silica and Carbon. Adsorption 2018, 24, 345-355.

https://doi.org/10.1007/s10450-018-9946-1.

(25) Calabrese, L.; Bonaccorsi, L.; Bruzzaniti, P.; Frazzica, A.; Freni, A.; Proverbio, E. Adsorption Performance and Thermodynamic Analysis of SAPO-34 Silicone Composite Foams for Adsorption Heat Pump Applications. Mater. Renew. Sustain. Energy 2018, 7 (4), 1-13.

https://doi.org/10.1007/s40243-018-0131-y. 